\title{
A Class of Nonlinear Nonglobal Semi-Jordan Triple Derivable Mappings on Triangular Algebras
}

\author{
Xiuhai Fei (iD) and Haifang Zhang (ii) \\ School of Mathematics and Physics, West Yunnan University, Lingcang 677099, China \\ Correspondence should be addressed to Haifang Zhang; 907427798@qq.com
}

Received 27 August 2021; Accepted 14 September 2021; Published 25 September 2021

Academic Editor: Wenpeng Zhang

Copyright (C) 2021 Xiuhai Fei and Haifang Zhang. This is an open access article distributed under the Creative Commons Attribution License, which permits unrestricted use, distribution, and reproduction in any medium, provided the original work is properly cited.

In this paper, we proved that each nonlinear nonglobal semi-Jordan triple derivable mapping on a 2-torsion free triangular algebra is an additive derivation. As its application, we get the similar conclusion on a nest algebra or a 2-torsion free block upper triangular matrix algebra, respectively.

\section{Introduction}

Let $R$ be a commutative ring with identity and $A$ a unital algebra over $R, \Omega=\left\{X \in A: X^{2}=0\right\}$, and $\Delta$ be an additive mapping on $A$. For any $X, Y \in A$, we denote the Jordan product of $X, Y$ by $X^{\circ} Y=X Y+Y X$. For any $X \in A$, if $2 X=$ 0 implies $X=0$, then $A$ is said a 2-torsion free algebra. Recall that $\Delta$ is called a derivation if $\Delta(X Y)=\Delta(X) Y+$ $X \Delta(Y)$ for all $X, Y \in A ; \Delta$ is called a Jordan derivation if $\Delta\left(X^{\circ} \mathrm{Y}\right)=\Delta(\mathrm{X})^{\circ} \mathrm{Y}+\mathrm{X}^{\circ} \Delta(\mathrm{Y})$, for all $X, Y \in A ; \Delta$ is called a triple derivation if $\Delta(X Y Z)=\Delta(X) Y Z+X \quad \Delta(Y) Z+$ $X Y \Delta(Z)$, for all $X, Y, Z \in A . \Delta$ is called a Jordan triple derivation if $\Delta\left(X^{\circ} Y^{\circ} \mathrm{Z}\right)=\Delta(\mathrm{X})^{\circ} \mathrm{Y}^{\circ} \mathrm{Z}+\mathrm{X}^{\circ} \Delta(\mathrm{Y})^{\circ} \mathrm{Z}+\mathrm{X}^{\circ} \mathrm{Y}^{\circ}$ $\Delta(Z)$, for all $X, Y, Z \in A$. Furthermore, if $\Delta$ is without assumption of additivity in the above definitions, then $\Delta$ is said a nonlinear (triple) derivable mapping and a nonlinear Jordan (triple) derivable mapping, respectively. Obviously, every derivation is a Jordan derivation, every derivation is a triple derivation, and every triple derivation is a Jordan triple derivation. However, the inverse statement is not true in general.

A natural and very interesting problem that we are dealing with is studying certain conditions on an algebra such that each Jordan (triple) derivation (nonlinear Jordan (triple) derivable mapping) is a derivation.
In the past few decades, many mathematicians studied this problem and obtained abundant results. For example, Herstein, in [1], proved that every Jordan derivation on a prime ring not of characteristic 2 is a derivation. This result was extended by Cusack in [2] and Brešar and Vukman in [3] to the case of semiprime, respectively. Zhang, in [4, 5], showed that every Jordan derivation on a nest algebra or a 2torsion free triangular algebra is an inner derivation or a derivation, respectively. Later, Ghahramani, in [6], extended the result of Zhang and $\mathrm{Yu}$ in [5] and proved that, under certain conditions, each Jordan derivation on trivial extension algebras is a sum of a derivation and an antiderivation. For other similar results about Jordan derivations (nonlinear Jordan derivable mappings), we refer the readers to [7-9] and references therein, for more details. With the deepening of research, many research achievements have been obtained about Jordan triple derivations and nonlinear Jordan triple derivable mappings. For example, Bresar, in [10], proved that every Jordan triple derivation on a 2-torsion-free semiprime ring is a derivation. Similar conclusion have been obtained in [11] by Bell and Kappe. Zhao and Li, in [12], proved that every nonlinear * -Jordan triple derivation on von Neumann algebras is an additive $*$-derivation. For other similar results about Jordan triple derivations (nonlinear Jordan triple derivable 
mappings), we refer the readers to [13-15] and references therein, for more details.

In 2016, Ashraf and Jabeen, in [15], obtained that if $\Delta$ is without the additivity assumption and satisfies

$$
\begin{aligned}
\Delta(X Y Z+Z Y X)= & \Delta(X) Y Z+X \Delta(Y) Z+X Y \Delta(Z) \\
& +\Delta(Z) Y X+Z \Delta(Y) X+Z Y \Delta(X),
\end{aligned}
$$

for all $X, Y, Z \in A$, then such a $\Delta$ is an additive derivation on a 2-torsion-free triangular algebra.

In this paper, we call that $\Delta$ is a nonlinear nonglobal semi-Jordan triple derivable mapping on $A$ if $\Delta$ is without the additivity assumption and satisfies

$$
\begin{aligned}
\Delta(X Y Z+Y X Z)= & \Delta(X) Y Z+X \Delta(Y) Z+X Y \Delta(Z) \\
& +\Delta(Y) X Z+Y \Delta(X) Z+Y X \Delta(Z),
\end{aligned}
$$

for all $X, Y, Z \in A$ with $X Y Z \in \Omega$.

Here, it needs to be pointed out that our above definition is different from Ashraf's and Jabeen's in [15]. We will discuss the nonlinear nonglobal semi-Jordan triple derivable mappings on triangular algebras and obtain one main result (see Theorem 1).

For convenient reading, we give some basic concepts and properties of triangular algebras as follows.

Let $A$ and $B$ be unital algebras over a commutative ring $R$ and $M$ be a unital $(A, B)$-bimodule, which is faithful as both are a left $A$-module and a right $B$-module. Then, the $R$-algebra,

$$
U=\left\{\left(\begin{array}{cc}
a & m \\
0 & b
\end{array}\right): \quad a \in A, m \in M, b \in B\right\},
$$

under the usual matrix operations is called a triangular algebra. We refer the reader to [16] for more details about the triangular algebras. Basic examples of triangular algebras are upper triangular matrix algebras and nest algebras.

Let $1_{A}$ and $1_{B}$ be the identities of the algebras $A$ and $B$, respectively, and let 1 be the identity of the triangular algebra $U$. Throughout this paper, we shall use the following notations:

$$
\begin{aligned}
& P_{1}=\left(\begin{array}{cc}
1_{A} & 0 \\
0 & 0
\end{array}\right), \\
& P_{2}=1-P_{1}=\left(\begin{array}{cc}
0 & 0 \\
0 & 1_{B}
\end{array}\right) .
\end{aligned}
$$

It is clear that the triangular algebra $U$ may be represented as

$$
U=P_{1} U P_{1}+P_{1} U P_{2}+P_{2} U P_{2}=A+M+B
$$

where $P_{1} U P_{1}$ and $P_{2} U P_{2}$ are subalgebras of $U$ isomorphic to $A$ and $B$, respectively, and $P_{1} U P_{2}$ is a $\left(P_{1} U P_{1}\right.$, $\left.P_{2} U P_{2}\right)$-bimodule isomorphic to the $(A, B)$-bimodule $M$.

\section{Nonlinear Nonglobal Semi-Jordan Triple Derivable Mappings on Triangular Algebras}

In this section, our main result is Theorem 1, and we will show Theorem 1 holds.

Theorem 1. Let $U$ be a 2-torsion-free triangular algebra and $\Delta$ be a mapping from $U$ into itself (without assumption of additivity) such that

$$
\begin{aligned}
\Delta(X Y Z+Y X Z)= & \Delta(X) Y Z+X \Delta(Y) Z+X Y \Delta(Z)+ \\
& \Delta(Y) X Z+Y \Delta(X) Z+Y X \Delta(Z),
\end{aligned}
$$

for all $X, Y, Z \in U$ with $X Y Z \in \Omega$. Then, $\Delta$ is an additive derivation.

In order to prove Theorem 1, we introduce Lemmas 1-5 and then prove that Lemmas 1-5 hold. We assume that $U$ be a 2-torsion-free triangular algebra $\Omega=\left\{X \in U: X^{2}=0\right\}$, and $\Delta$ be a nonlinear nonglobal semi-Jordan triple derivable mapping on triangular algebra $U$.

Lemma 1. $\Delta(0)=0$ and $\Delta\left(P_{1}\right)=-\Delta\left(P_{2}\right) \in M$.

Proof. Taking $X=Y=Z=0$ in equation (6), we have $\Delta(0)=0$. Since $P_{1} P_{1} P_{2}=0 \in \Omega$, taking $X=P_{1}, Y=P_{1}, Z=$ $P_{2}$ in equation (6), we obtain

$$
0=\Delta(0)=2 \Delta\left(P_{1}\right) P_{1} P_{2}+2 P_{1} \Delta\left(P_{1}\right) P_{2}+2 P_{1} P_{1} \Delta\left(P_{2}\right)=2 P_{1} \Delta\left(P_{1}\right) P_{2}+2 P_{1} \Delta\left(P_{2}\right)
$$



that

This yields from the property of 2-torsion freeness of $U$

$$
\begin{aligned}
P_{1} \Delta\left(P_{2}\right) P_{1} & =0, \\
P_{1} \Delta\left(P_{1}\right) P_{2}+P_{1} \Delta\left(P_{2}\right) P_{2} & =0 .
\end{aligned}
$$

Similarly, we obtain that

$$
P_{2} \Delta\left(P_{1}\right) P_{2}=0 .
$$

For any $X_{12} \in M$, since $P_{2} X_{12} P_{2}=0 \in \Omega$, taking $X=$ $P_{2}, Y=X_{12}$, and $Z=P_{2}$ in equation (6), we have

$$
\begin{aligned}
\Delta\left(X_{12}\right)= & \Delta\left(P_{2} X_{12} P_{2}+X_{12} P_{2} P_{2}\right) \\
= & \Delta\left(P_{2}\right) X_{12} P_{2}+P_{2} \Delta\left(X_{12}\right) P_{2}+P_{2} X_{12} \Delta\left(P_{2}\right) \\
& +\Delta\left(X_{12}\right) P_{2} P_{2}+X_{12} \Delta\left(P_{2}\right) P_{2}+X_{12} P_{2} \Delta\left(P_{2}\right) \\
= & \Delta\left(P_{2}\right) X_{12}+P_{2} \Delta\left(X_{12}\right) P_{2}+\Delta\left(X_{12}\right) P_{2}+X_{12} \Delta\left(P_{2}\right) P_{2}+X_{12} P_{2} \Delta\left(P_{2}\right) .
\end{aligned}
$$

Multiplying the above equation from left by $P_{1}$ and from right by $P_{2}$, it follows from $P_{1} \Delta\left(P_{2}\right) P_{1}=0$ that $2 X_{12} P_{2} \Delta\left(P_{2}\right) P_{2}=0$. Similarly, we obtain that $2 P_{1} \Delta\left(P_{2}\right)$ $P_{1} X_{12}=0$. Therefore, according to the faithfulness of $M$ and the property of 2-torsion free of $U$, we have

$$
P_{1} \Delta\left(P_{1}\right) P_{1}=P_{2} \Delta\left(P_{2}\right) P_{2}=0 .
$$

So, by equations (8)-(11), we have $\Delta\left(P_{1}\right)=-\Delta\left(P_{2}\right)$ $\in M$. The proof is completed.

Lemma 2. For any $X_{11} \in A, X_{12} \in M, X_{22} \in B$, then

(i) $\Delta\left(X_{11}\right) \in A+M$ and $P_{1} \Delta\left(X_{11}\right) P_{2}=X_{11} \Delta\left(P_{1}\right)$

(ii) $\Delta\left(X_{22}\right) \in M+B$ and $P_{1} \Delta\left(X_{22}\right) P_{2}=\Delta\left(P_{2}\right) X_{22}$

(iii) $\Delta\left(X_{12}\right) \in M$

Proof. (i) For any $X_{11} \in A$, since $X_{11} P_{2} P_{2}=0 \in \Omega$, taking $X=X_{11}, Y=Z=P_{2}$ in equation (6), then it follows from $\Delta\left(P_{2}\right) \in M$ that

$$
\begin{aligned}
0= & \Delta\left(X_{11} P_{2} P_{2}+P_{2} X_{11} P_{2}\right), \\
= & \Delta\left(X_{11}\right) P_{2} P_{2}+X_{11} \Delta\left(P_{2}\right) P_{2} \\
& +X_{11} P_{2} \Delta\left(P_{2}\right)+\Delta\left(P_{2}\right) X_{11} P_{2}+P_{2} \Delta\left(X_{11}\right) P_{2}+P_{2} X_{11} \Delta\left(P_{2}\right), \\
= & \Delta\left(X_{11}\right) P_{2}+X_{11} \Delta\left(P_{2}\right) P_{2}+P_{2} \Delta\left(X_{11}\right) P_{2} .
\end{aligned}
$$

This implies that $P_{2} \Delta\left(X_{11}\right) P_{2}=0$. Furthermore, multiplying the above equation from left by $P_{1}$ and from right by $P_{2}$, it follows from $\Delta\left(P_{1}\right)=-\Delta\left(P_{2}\right) \in M$ that

$$
P_{1} \Delta\left(X_{11}\right) P_{2}=-X_{11} \Delta\left(P_{2}\right)=X_{11} \Delta\left(P_{1}\right) .
$$

Similarly, we can show (ii) holds.

(iii) For any $X_{12} \in M$, since $X_{12} P_{1} P_{2}=0 \in \Omega$, taking $X=X_{12}, Y=P_{1}$, and $Z=P_{2}$ equation (6), we get from $\Delta\left(P_{1}\right)=-\Delta\left(P_{2}\right) \in M$ that

$$
\begin{aligned}
\Delta\left(X_{12}\right)= & \Delta\left(X_{12} P_{1} P_{2}+P_{1} X_{12} P_{2}\right) \\
& +\Delta\left(X_{12}\right) P_{1} P_{2}+X_{12} \Delta\left(P_{1}\right) P_{2}+X_{12} P_{1} \Delta\left(P_{2}\right) \\
& +\Delta\left(P_{1}\right) X_{12} P_{2}+P_{1} \Delta\left(X_{12}\right) P_{2}+P_{1} X_{12} \Delta\left(P_{2}\right) \\
= & P_{1} \Delta\left(X_{12}\right) P_{2} .
\end{aligned}
$$

Therefore, $\Delta\left(X_{12}\right) \in M$. The proof is completed.

Lemma 3. For any $X_{11}, Y_{11} \in A, \quad X_{12} \in M$, and $X_{22}, Y_{22} \in B$, then
(i) $\Delta\left(X_{11} X_{22}\right)=\Delta\left(X_{11}\right) X_{22}+X_{11} \Delta\left(X_{22}\right)$
(ii) $\Delta\left(X_{11} X_{12}\right)=\Delta\left(X_{11}\right) X_{12}+X_{11} \Delta\left(X_{12}\right)$
(iii) $\Delta\left(X_{12} X_{22}\right)=\Delta\left(X_{12}\right) X_{22}+X_{12} \Delta\left(X_{22}\right)$
(iv) $\Delta\left(X_{11} Y_{11}\right)=\Delta\left(X_{11}\right) Y_{11}+X_{11} \Delta\left(Y_{11}\right)$
(v) $\Delta\left(X_{22} Y_{22}\right)=\Delta\left(X_{22}\right) Y_{22}+X_{22} \Delta\left(Y_{22}\right)$

Proof. (i) For any $X_{11} \in A$ and $X_{22} \in B$, since $X_{11} X_{22} P_{2}=$ $0 \in \Omega$, taking $X=X_{11}, Y=X_{22}$, and $Z=P_{2}$ in equation (6), it follows from Lemma 2 that

$$
\begin{aligned}
0= & \Delta\left(X_{11} X_{22}\right)=\Delta\left(X_{11} X_{22} P_{2}+X_{22} X_{11} P_{2}\right) \\
= & \Delta\left(X_{11}\right) X_{22} P_{2}+X_{11} \Delta\left(X_{22}\right) P_{2}+X_{11} X_{22} \Delta\left(P_{2}\right) \\
& +\Delta\left(X_{22}\right) X_{11} P_{2}+X_{22} \Delta\left(X_{11}\right) P_{2}+X_{22} X_{11} \Delta\left(P_{2}\right) \\
= & \Delta\left(X_{11}\right) X_{22}+X_{11} \Delta\left(X_{22}\right) .
\end{aligned}
$$

(ii) For any $X_{11} \in A$ and $X_{12} \in M$, since $X_{12} X_{11} P_{2}=0 \in \Omega$, taking $X=X_{12}, Y=X_{11}$, and $Z=P_{2}$ in equation (6), we can obtain from $\Delta\left(P_{2}\right) \in M$ and Lemma 2 that

$$
\begin{aligned}
\Delta\left(X_{11} X_{12}\right)= & \Delta\left(X_{12} X_{11} P_{2}+X_{11} X_{12} P_{2}\right) \\
= & \Delta\left(X_{12}\right) X_{11} P_{2}+X_{12} \Delta\left(X_{11}\right) P_{2}+X_{12} X_{11} \Delta\left(P_{2}\right) \\
& +\Delta\left(X_{11}\right) X_{12} P_{2}+X_{11} \Delta\left(X_{12}\right) P_{2}+X_{11} X_{12} \Delta\left(P_{2}\right) \\
= & \Delta\left(X_{11}\right) X_{12}+X_{11} \Delta\left(X_{12}\right) .
\end{aligned}
$$

Similarly, we can show (iii) holds.

(iv) For any $X_{11}, Y_{11} \in A$ and $Z_{12} \in M$, by Lemma 2 (ii), on the one hand, we get that

$$
\begin{aligned}
\Delta\left(X_{11} Y_{11} Z_{12}\right)= & \Delta\left(\left(X_{11} Y_{11}\right) Z_{12}\right)=\Delta\left(X_{11} Y_{11}\right) Z_{12} \\
& +X_{11} Y_{11} \Delta\left(Z_{12}\right) .
\end{aligned}
$$

On the other hand, 


$$
\begin{aligned}
\Delta\left(X_{11} Y_{11} Z_{12}\right)= & \Delta\left(X_{11}\left(Y_{11} Z_{12}\right)\right)=\Delta\left(X_{11}\right) Y_{11} Z_{12} \\
& +X_{11} \Delta\left(Y_{11}\right) Z_{12}+X_{11} Y_{11} \Delta\left(Z_{12}\right) .
\end{aligned}
$$

Comparing the above two equations, we obtain

$$
\left(\Delta\left(X_{11} Y_{11}\right)-\Delta\left(X_{11}\right) Y_{11}-X_{11} \Delta\left(Y_{11}\right)\right) Z_{12}=0 .
$$

This yields from the faithfulness of $M$ and Lemma 2 that

$$
\begin{aligned}
P_{1} \Delta\left(X_{11} Y_{11}\right) P_{1}= & P_{1} \Delta\left(X_{11}\right) Y_{11}+X_{11} \Delta\left(Y_{11}\right) P_{1}=\Delta\left(X_{11}\right) Y_{11} \\
& +X_{11} \Delta\left(Y_{11}\right) P_{1} .
\end{aligned}
$$

Furthermore, by Lemma 2 (i), we obtain that

$$
P_{1} \Delta\left(X_{11} Y_{11}\right) P_{2}=X_{11} Y_{11} \Delta\left(P_{1}\right)=X_{11} \Delta\left(Y_{11}\right) P_{2} \text {. }
$$

Therefore, by the above two equations and $P_{2} \Delta\left(X_{11} Y_{11}\right) P_{2}=0$, we get $\Delta\left(X_{11} Y_{11}\right)=\Delta\left(X_{11}\right) Y_{11}+$ $X_{11} \Delta\left(Y_{11}\right)$. Similarly, we can show $(v)$ holds. The proof is completed.

Lemma 4. For any $X_{11}, Y_{11} \in A, \quad X_{12}, Y_{12} \in M$, and $X_{22}, Y_{22} \in B$, then
(i) $\Delta\left(X_{11}+X_{12}\right)=\Delta\left(X_{11}\right)+\Delta\left(X_{12}\right)$
(ii) $\Delta\left(X_{12}+X_{22}\right)=\Delta\left(X_{12}\right)+\Delta\left(X_{22}\right)$
(iii) $\Delta\left(X_{12}+Y_{12}\right)=\Delta\left(X_{12}\right)+\Delta\left(Y_{12}\right)$
(iv) $\Delta\left(X_{11}+Y_{11}\right)=\Delta\left(X_{11}\right)+\Delta\left(Y_{11}\right)$
(v) $\Delta\left(X_{22}+Y_{22}\right)=\Delta\left(X_{22}\right)+\Delta\left(Y_{22}\right)$

Proof. (i) For any $X_{11} \in A, X_{12}, Y_{12} \in M$, and $X_{22} \in B$, since $P_{2}\left(X_{11}+X_{12}\right) P_{2}=0 \in \Omega$, taking $X=P_{2}, Y=X_{11}+$ $X_{12}$, and $Z=P_{2}$ in equation (6), then by $\Delta\left(P_{2}\right) \in M$ and $P_{1} \Delta\left(X_{11}\right) P_{2}=-X_{11} \Delta\left(P_{2}\right)$, we obtain

$$
\begin{aligned}
\Delta\left(X_{12}\right)= & \Delta\left(P_{2}\left(X_{11}+X_{12}\right) P_{2}+\left(X_{11}+X_{12}\right) P_{2} P_{2}\right) \\
= & \Delta\left(P_{2}\right)\left(X_{11}+X_{12}\right) P_{2}+P_{2} \Delta\left(X_{11}+X_{12}\right) P_{2}+P_{2}\left(X_{11}+X_{12}\right) \Delta\left(P_{2}\right) \\
& +\Delta\left(X_{11}+X_{12}\right) P_{2} P_{2}+\left(X_{11}+X_{12}\right) \Delta\left(P_{2}\right) P_{2}+\left(X_{11}+X_{12}\right) P_{2} \Delta\left(P_{2}\right) \\
= & P_{2} \Delta\left(X_{11}+X_{12}\right) P_{2}+\Delta\left(X_{11}+X_{12}\right) P_{2}+X_{11} \Delta\left(P_{2}\right) P_{2} \\
= & P_{2} \Delta\left(X_{11}+X_{12}\right) P_{2}+\Delta\left(X_{11}+X_{12}\right) P_{2}-P_{1} \Delta\left(X_{11}\right) P_{2} .
\end{aligned}
$$

Then, it follows from $\Delta\left(X_{12}\right)=P_{1} \Delta\left(X_{12}\right) P_{2}$ and the property of 2-torsion freeness of $U$ that

$$
\begin{aligned}
& P_{2} \Delta\left(X_{11}+X_{12}\right) P_{2}=0, \\
& P_{1} \Delta\left(X_{11}+X_{12}\right) P_{2}=P_{1} \Delta\left(X_{11}\right) P_{2}+\Delta\left(X_{12}\right) .
\end{aligned}
$$

Furthermore, since $Y_{12}\left(X_{11}+X_{12}\right) P_{2}=0 \in \Omega$, taking $X=Y_{12}, Y=X_{11}+X_{12}$, and $Z=P_{2}$ in equation (6), then we obtain from Lemma $2, \quad \Delta\left(P_{2}\right) \in M$, and $P_{2} \Delta\left(X_{11}+X_{12}\right) P_{2}=0$ that

$$
\begin{aligned}
\Delta\left(X_{11} Y_{12}\right)= & \Delta\left(Y_{12}\left(X_{11}+X_{12}\right) P_{2}+\left(X_{11}+X_{12}\right) Y_{12} P_{2}\right) \\
= & \Delta\left(Y_{12}\right)\left(X_{11}+X_{12}\right) P_{2}+Y_{12} \Delta\left(X_{11}+X_{12}\right) P_{2}+Y_{12}\left(X_{11}+X_{12}\right) \Delta\left(P_{2}\right) \\
& +\Delta\left(X_{11}+X_{12}\right) Y_{12} P_{2}+\left(X_{11}+X_{12}\right) \Delta\left(Y_{12}\right) P_{2}+\left(X_{11}+X_{12}\right) Y_{12} \Delta\left(P_{2}\right) \\
= & \Delta\left(X_{11}+X_{12}\right) Y_{12}+X_{11} \Delta\left(Y_{12}\right) .
\end{aligned}
$$

This yields from Lemma 3 (ii) that $\left(\Delta\left(X_{11}+X_{12}\right)-\Delta\left(X_{11}\right)\right) Y_{12}=0$, and then, by the faithfulness of $M$, we get that

$$
P_{1} \Delta\left(X_{11}+X_{12}\right) P_{1}=P_{1} \Delta\left(X_{11}\right) P_{1} .
$$

Hence, by equations (23)-(31) and Lemma 2, we get $\Delta\left(X_{11}+X_{12}\right)=\Delta\left(X_{11}\right)+\Delta\left(X_{12}\right)$. Similarly, we can show (ii) holds.

(iii) For any $X_{12}, Y_{12} \in M$, since $\left(P_{1}+X_{12}\right)\left(Y_{12}+P_{2}\right) P_{2}=X_{12}+Y_{12} \in \Omega$, taking $X=\left(P_{1}+\right.$ 
$\left.X_{12}\right), Y=\left(P_{2}+Y_{12}\right)$, and $Z=P_{2}$ in equation (6), then by Lemmas 2 and 4 (i)-(ii) and $\Delta\left(P_{1}\right)=-\Delta\left(P_{2}\right) \in M$, we get that

$$
\begin{aligned}
\Delta\left(X_{12}+Y_{12}\right)= & \Delta\left(\left(P_{1}+X_{12}\right)\left(Y_{12}+P_{2}\right) P_{2}+\left(Y_{12}+P_{2}\right)\left(P_{1}+X_{12}\right) P_{2}\right) \\
= & \Delta\left(P_{1}+X_{12}\right)\left(Y_{12}+P_{2}\right) P_{2}+\left(P_{1}+X_{12}\right) \Delta\left(Y_{12}+P_{2}\right) P_{2} \\
& +\left(P_{1}+X_{12}\right)\left(Y_{12}+P_{2}\right) \Delta\left(P_{2}\right) \\
& +\Delta\left(Y_{12}+P_{2}\right)\left(P_{1}+X_{12}\right) P_{2}+\left(Y_{12}+P_{2}\right) \Delta\left(P_{1}+X_{12}\right) P_{2} \\
& +\left(Y_{12}+P_{2}\right)\left(P_{1}+X_{12}\right) \Delta\left(P_{2}\right) \\
= & \Delta\left(P_{1}\right)+\Delta\left(X_{12}\right)+\Delta\left(Y_{12}\right)+\Delta\left(P_{2}\right) \\
= & \Delta\left(X_{12}\right)+\Delta\left(Y_{12}\right) .
\end{aligned}
$$

(iv) For any $X_{11}, Y_{11} \in A$ and $Z_{12} \in M$, since $Z_{12}\left(X_{11}+Y_{11}\right) P_{2}=0 \in \Omega$, taking $X=Z_{12}, Y=\left(X_{11}+\right.$
$\left.Y_{11}\right)$, and $Z=P_{2}$ in equation (6), then it follows from $\Delta\left(P_{2}\right) \in M$ that

$$
\begin{aligned}
\Delta\left(X_{11} Z_{12}+Y_{11} Z_{12}\right)= & \Delta\left(Z_{12}\left(X_{11}+Y_{11}\right) P_{2}+\left(X_{11}+Y_{11}\right) Z_{12} P_{2}\right) \\
= & \Delta\left(Z_{12}\right)\left(X_{11}+Y_{11}\right) P_{2}+Z_{12} \Delta\left(X_{11}+Y_{11}\right) P_{2}+Z_{12}\left(X_{11}+Y_{11}\right) \Delta\left(P_{2}\right) \\
& +\Delta\left(X_{11}+Y_{11}\right) Z_{12} P_{2}+\left(X_{11}+Y_{11}\right) \Delta\left(Z_{12}\right) P_{2}+\left(X_{11}+Y_{11}\right) Z_{12} \Delta\left(P_{2}\right) \\
= & \Delta\left(X_{11}+Y_{11}\right) Z_{12}+\left(X_{11}+Y_{11}\right) \Delta\left(Z_{12}\right) .
\end{aligned}
$$

Therefore, this implies from Lemmas 4 (iii) and 3 (ii) that $\left(\Delta\left(X_{11}+Y_{11}\right)-\Delta\left(X_{11}\right)-\Delta\left(Y_{11}\right)\right) Z_{12}=0$, so by the faithfulness of $M$, we get that

$$
P_{1} \Delta\left(X_{11}+Y_{11}\right) P_{1}=P_{1} \Delta\left(X_{11}\right) P_{1}+P_{1} \Delta\left(Y_{11}\right) P_{1} .
$$

Furthermore, for any $X_{11}, Y_{11} \in A$, we can get from Lemma 2 (i) that

$$
\begin{aligned}
P_{1} \Delta\left(X_{11}+Y_{11}\right) P_{2}= & \left(X_{11}+Y_{11}\right) \Delta\left(P_{1}\right)=X_{11} \Delta\left(P_{1}\right) \\
& +Y_{11} \Delta\left(P_{1}\right)=P_{1} \Delta\left(X_{11}\right) P_{2}+P_{1} \Delta\left(Y_{11}\right) P_{2} .
\end{aligned}
$$

Therefore, we get from above two equations and $P_{2} \Delta\left(X_{11}+Y_{11}\right) P_{2}=0$

that $\Delta\left(X_{11}+Y_{11}\right)=\Delta\left(X_{11}\right)+\Delta\left(Y_{11}\right)$. Similarly, we can show $(v)$ holds. The proof is completed.

Lemma 5. For any $X_{11} \in A, X_{12} \in M$, and $X_{22} \in B$, then $\Delta\left(X_{11}+X_{12}+X_{22}\right)=\Delta\left(X_{11}\right)+\Delta\left(X_{12}\right)+\Delta\left(X_{22}\right)$.

Proof. For any $X_{11} \in A, X_{12} \in M$, and $X_{22} \in B$, since $P_{1}\left(X_{11}+X_{12}+X_{22}\right) P_{2}=X_{12} \in \Omega$, taking $X=P_{1}, Y=X_{11}$ $+X_{12}+X_{22}, Y=P_{1}$, and $Z=P_{2}$ in equation (6), then it follows from $\Delta\left(P_{1}\right)=-\Delta\left(P_{2}\right) \in M$ and Lemma 2 that

$$
\begin{aligned}
\Delta\left(X_{12}\right)= & \Delta\left(P_{1}\left(X_{11}+X_{12}+X_{22}\right) P_{2}+\left(X_{11}+X_{12}+X_{22}\right) P_{1} P_{2}\right) \\
= & \Delta\left(P_{1}\right)\left(X_{11}+X_{12}+X_{22}\right) P_{2}+P_{1} \Delta\left(X_{11}+X_{12}+X_{22}\right) P_{2}+P_{1}\left(X_{11}+X_{12}+X_{22}\right) \Delta\left(P_{2}\right) \\
& +\Delta\left(X_{11}+X_{12}+X_{22}\right) P_{1} P_{2}+\left(X_{11}+X_{12}+X_{22}\right) \Delta\left(P_{1}\right) P_{2}+\left(X_{11}+X_{12}+X_{22}\right) P_{1} \Delta\left(P_{2}\right) \\
= & \Delta\left(P_{1}\right) X_{22}+P_{1} \Delta\left(X_{11}+X_{12}+X_{22}\right) P_{2}+X_{11} \Delta\left(P_{2}\right)+X_{11} \Delta\left(P_{1}\right)+X_{11} \Delta\left(P_{2}\right) \\
= & P_{1} \Delta\left(X_{11}+X_{12}+X_{22}\right) P_{2}-P_{1} \Delta\left(X_{11}\right) P_{2}-P_{1} \Delta\left(X_{22}\right) P_{2} .
\end{aligned}
$$


Hence, we get that

$$
\begin{aligned}
P_{1} \Delta\left(X_{11}+X_{12}+X_{22}\right) P_{2}= & P_{1} \Delta\left(X_{11}\right) P_{2}+\Delta\left(X_{12}\right) \\
& +P_{1} \Delta\left(X_{22}\right) P_{2} .
\end{aligned}
$$

In the following, we will show that $P_{1} \Delta\left(X_{11}+X_{12}+\right.$ $\left.X_{22}\right) P_{1}=P_{1} \Delta\left(X_{11}\right) P_{1}$
$P_{2} \Delta\left(X_{11}+X_{12}+X_{22}\right) P_{2}=P_{2} \Delta\left(X_{22}\right) P_{2}$. Indeed, for any $Z_{12} \in M$, since $P_{1}\left(X_{11}+X_{12}+X_{22}\right) Z_{12}=X_{11} Z_{12} \in \Omega$, taking $X=P_{1}, Y=X_{11}+X_{12}+X_{22}$, and $Z=Z_{12}$ in equation (6), then, by Lemma 2 and $\Delta\left(P_{1}\right) \in M$, we get that

$$
\begin{aligned}
\Delta\left(2 X_{11} Z_{12}\right)= & \Delta\left(P_{1}\left(X_{11}+X_{12}+X_{22}\right) Z_{12}+\left(X_{11}+X_{12}+X_{22}\right) P_{1} Z_{12}\right) \\
= & \Delta\left(P_{1}\right)\left(X_{11}+X_{12}+X_{22}\right) Z_{12}+P_{1} \Delta\left(X_{11}+X_{12}+X_{22}\right) Z_{12} \\
& +P_{1}\left(X_{11}+X_{12}+X_{22}\right) \Delta\left(Z_{12}\right) \\
& +\Delta\left(X_{11}+X_{12}+X_{22}\right) P_{1} Z_{12}+\left(X_{11}+X_{12}+X_{22}\right) \Delta\left(P_{1}\right) Z_{12} \\
& +\left(X_{11}+X_{12}+X_{22}\right) P_{1} \Delta\left(Z_{12}\right) \\
= & 2 P_{1} \Delta\left(X_{11}+X_{12}+X_{22}\right) Z_{12}+2 X_{11} \Delta\left(Z_{12}\right) .
\end{aligned}
$$

On the contrary, it follows from Lemma 4 (iii) and Lemma 3 (ii) that

$$
\Delta\left(2 X_{11} Z_{12}\right)=2 \Delta\left(X_{11} Z_{12}\right)=2 \Delta\left(X_{11}\right) Z_{12}+2 X_{11} \Delta\left(Z_{12}\right) \text {. }
$$

Comparing the above two equations, we get that $2 P_{1}\left(\Delta\left(X_{11}+X_{12}+X_{22}\right)-\Delta\left(X_{11}\right)\right) P_{1} Z_{12}=0$, and then, by the faithfulness of $M$ and the property of 2-torsion free of $U$, we have

$$
P_{1} \Delta\left(X_{11}+X_{12}+X_{22}\right) P_{1}=P_{1} \Delta\left(X_{11}\right) P_{1} .
$$

Similarly, we can obtain that

$$
P_{2} \Delta\left(X_{11}+X_{12}+X_{22}\right) P_{2}=P_{2} \Delta\left(X_{22}\right) P_{2} .
$$

Therefore, by equations (31)-(35), we get $\Delta\left(X_{11}+X_{12}\right.$ $\left.+X_{22}\right)=\Delta\left(X_{11}\right)+\Delta\left(X_{12}\right)+\Delta\left(X_{22}\right)$. The proof is completed.

In the following, we give the completed proof of Theorem 1.

Proof of Theorem 1. It follows from Lemmas 4 and 5 that $\Delta$ is an additive mapping on $U$. Next, we show that $\Delta$ is an derivation on $U$. Let $X=X_{11}+X_{12}+X_{22}$ and $Y=Y_{11}+$ $Y_{12}+Y_{22}$ be arbitrary elements of $U$, where $X_{11}, Y_{11} \in A, X_{12}, Y_{12} \in M$, and $X_{22}, Y_{22} \in B$. Since $\Delta$ is an additive mapping on $U$, then it follows from Lemmas 1-3 that

$$
\begin{aligned}
\Delta(X Y)= & \Delta\left(X_{11} Y_{11}+X_{11} Y_{12}+X_{12} Y_{22}+X_{22} Y_{22}\right) \\
= & \Delta\left(X_{11} Y_{11}\right)+\Delta\left(X_{11} Y_{12}\right)+\Delta\left(X_{12} Y_{22}\right)+\Delta\left(X_{22} Y_{22}\right) \\
= & \Delta\left(X_{11}\right) Y_{11}+X_{11} \Delta\left(Y_{11}\right)+\Delta\left(X_{11}\right) Y_{12}+X_{11} \Delta\left(Y_{12}\right) \\
& +\Delta\left(X_{12}\right) Y_{22}+X_{12} \Delta\left(Y_{22}\right)+\Delta\left(X_{22}\right) Y_{22}+X_{22} \Delta\left(Y_{22}\right) \\
= & \Delta\left(X_{11}+X_{12}+X_{22}\right)\left(Y_{11}+Y_{12}+Y_{22}\right)+\left(X_{11}+X_{12}+X_{22}\right) \Delta\left(Y_{11}+Y_{12}+Y_{22}\right) \\
= & \Delta(X) Y+X \Delta(Y) .
\end{aligned}
$$

Therefore, $\Delta$ is an additive derivation on $U$. The proof is completed.

Next, we give an application of Theorem 1 to certain special classes of triangular algebras, such as block upper triangular matrix algebras and nest algebras.

Let $R$ be a commutative ring with identity, and let $M_{n \times k}(R)$ be the set of all $n \times k$ matrices over $R$. For $\underline{n} \geq 2$ and $m \leq n$, the block upper triangular matrix algebra $T_{n}^{\bar{k}}(R)$ is a subalgebra of $M_{n}(R)$ with the form

$$
\left(\begin{array}{cccc}
M_{k_{1}}(R) & M_{k_{1} \times k_{2}}(R) & \cdots & M_{k_{1} \times k_{m}}(R) \\
0 & M_{k_{2}}(R) & \cdots & M_{k_{2} \times k_{m}}(R) \\
\vdots & \vdots & \cdots & v \operatorname{dots} \\
0 & 0 & \cdots & M_{k_{m}}(R)
\end{array}\right),
$$

where $\bar{k}=\left(k_{1}, k_{2}, \ldots, k_{m}\right)$ is an ordered $m$-vector of positive integer such that $k_{1}+k_{2}+\cdots+k_{m}=n$.

A nest of a complex Hilbert space $H$ is a chain $\mathscr{N}$ of closed subspaces of $H$ containing $\{0\}$ and $H$ which is closed 
under arbitrary intersections and closed linear span. The nest algebra associated to $\mathcal{N}$ is the algebra:

$$
\operatorname{Alg} \mathcal{N}=\{T \in B(H): T N \subseteq N \quad \text { for all } N \in \mathscr{N}\} .
$$

A nest $\mathcal{N}$ is called trivial if $\mathcal{N}=\{0, H\}$. It is clear that every nontrivial nest algebra is a triangular algebra and every finite dimensional nest algebra is isomorphic to a complex block upper triangular matrix algebra.

Corollary 1. Let $T_{n}^{\bar{k}}(R)$ be a 2-torsion-free block upper triangular matrix algebra and $\Delta$ be a nonlinear nonglobal semiJordan triple derivable mapping on $T_{n}^{k}(R)$. Then, $\Delta$ is an additive derivation.

Corollary 2. Let $\mathcal{N}$ be a nontrivial nest of a complex Hilbert space $H, A \lg \mathcal{N}$ be a nest algebra, and $\Delta$ be a nonlinear nonglobal semi-Jordan triple derivable mapping on Alg $\mathcal{N}$. Then, $\Delta$ is an additive derivation.

\section{Data Availability}

No data were used to support the findings of the study.

\section{Conflicts of Interest}

The authors declare that they have no conflicts of interest.

\section{Authors' Contributions}

All authors contributed equally and significantly in this paper. All authors read and approved the final manuscript.

\section{Acknowledgments}

This work was supported by National Natural Science Foundation of China (no.11901451), Basic Research Foundation of Yunnan Education Department (nos.2020J0748 and 2021J0635), and Talent Project Foundation of Yunnan Provincial Science and Technology Department (no. 202105AC160089).

\section{References}

[1] I. N. Herstein, "Jordan derivations of prime rings," Proceedings of the American Mathematical Society, vol. 8, no. 6, p. 1104, 1957.

[2] J. M. Cusack, "Jordan derivations on rings," Proceedings of the American Mathematical Society, vol. 53, no. 2, p. 321, 1975.

[3] M. Brešar and J. Vukman, "Jordan derivations on semiprime rings," Bulletin of the Australian Mathematical Society, vol. 37, no. 3, pp. 321-322, 1988.

[4] J. H. Zhang, "Derivations on nest Algebras," Acta Mathematica Sinica, vol. 41, no. 1, pp. 205-212, 1998.

[5] J.-H. Zhang and W.-Y. Yu, "Jordan derivations of triangular algebras," Linear Algebra and Its Applications, vol. 419, no. 1, pp. 251-255, 2006.

[6] H. Ghahramani, "Jordan derivations on trivial extensions," Bulletin of the Iranian Mathematical Society, vol. 9, no. 4, pp. 635-645, 2013.
[7] G. Dolinar, K. He, B. Kuzma, and X. Qi, "A note on Jordan derivable linear maps," Operators and Matrices, vol. 7, no. 1, pp. 159-165, 2013.

[8] F. Lu, "Jordan derivable maps of prime rings," Communications in Algebra, vol. 38, no. 12, pp. 4430-4440, 2010.

[9] D. Benkovič, "Jordan derivations and antiderivations on triangular matrices," Linear Algebra and Its Applications, vol. 397, no. 11, pp. 235-244, 2005.

[10] M. Brešar, "Mapping of semiprime rings," Journal of Algebra, vol. 127, pp. 218-228, 1989.

[11] M. Foner and D. Ilievi, "On Jordan triple derivations and related mappings," Mediterranean Journal of Mathematics, vol. 5, pp. 415-C427, 2008.

[12] F. F. Zhao and C. J. Li, "Nonlinear *-Jordan triple derivations on von Neumann algebras," Mathematical Slovaca, vol. 1, pp. 163-170, 2018.

[13] H. E. Bell and L. C. Kappe, "Rings in which derivations satisfy certain algebraic conditions," Acta Mathematica Hungarica, vol. 53, no. 3-4, pp. 339-346, 1989.

[14] V. Darvish, M. Nourl, M. Razeghi, and A. Taghavi, "Nonlinear *-Jordan triple derivations on prime *-lagebras," 2019, https:// www.researchgate.net/publication/331485362.

[15] M. Ashraf and A. Jabeen, "Nonlinear Jordan triple derivable mappings of triangular algebras," Pacific Journal of Applied Mathematics, vol. 7, no. 4, pp. 229-239, 2016.

[16] W. S. Cheung, "Mappings on triangular algebras," Ph. D Thesis, University of Victoria, London, UK, 2000. 